\title{
No spin-glass transition in the "mobile-bond" model
}

\author{
A. K. Hartmann \\ Institut für Theoretische Physik, Universität Göttingen, Bunsenstraße 9, 37037 Göttingen, Germany
}

(Dated: February 7, 2020)

\begin{abstract}
The recently introduced "mobile-bond" model for two-dimensional spin glasses is studied. The model is characterized by an annealing temperature $T_{\mathrm{q}}$. On the basis of Monte Carlo simulations of small systems it has been claimed that this model exhibits a non-trivial spin-glass transition at finite temperature for small values of $T_{\mathrm{q}}$.

Here the model is studied by means of exact ground-state calculations of large systems up to $N=256^{2}$. The scaling of domain-wall energies is investigated as a function of the system size. For small values $T_{\mathrm{q}}<0.95$ the system behaves like a (gauge-transformed) ferromagnet having a small fraction of frustrated plaquettes. For $T_{\mathrm{q}} \geq 0.95$ the system behaves like the standard twodimensional $\pm J$ spin-glass, i.e. it does not exhibit a phase transition at $T>0$.

PACS numbers: 75.50.Lk, 05.70.Jk, 75.40.Mg, 77.80.Bh
\end{abstract}

Spin glasses 1 are the prototype model for disordered systems investigated extensively during the last three decades in statistical physics. These systems exhibit complex energy landscapes resulting in many interesting phenomena like glassy behavior and aging. Despite much effort, still many open questions exists. The question about the lower critical dimension of Ising spin glasses had been discussed for quite a while $e^{23.4 .5}$. Now it is clear that in two dimensions non stable spin-glass phase at finite temperature exists $6 \cdot 7 \cdot 8.9 .10$. This has motivated the search for other two-dimensional spin-glasslike systems exhibiting a $T_{\mathrm{c}}>0$ 11 . Recently D.K. Sunko has proposed"12 a "mobile-bond" model where quencheddisorder realizations of $\pm J$ spin glasses are created by an annealed simulation, allowing the bonds to move. The system is equilibrated at high temperature, followed by a quench to a temperature $T_{\mathrm{q}}$. Sunko has performed Monte-Carlo simulations of systems up to size $L=16$ and claimed that for low quenching temperatures $T_{\mathrm{q}}$ the model exhibits a spin-glass transition at finite temperature $T_{\mathrm{c}}>0$.

In this rapid communication, the model is studied by means of exact ground-state calculations of large systems up to $L=256$. The scaling of domain-wall energies s.3.13 $^{2.13}$ is studied as a function of the system size. It is shown here that the model exhibits no spin-glass transition at finite temperature. For small values of $T_{\mathrm{q}}<0.95$ the system exhibits ferromagnetic order, while at $T_{\mathrm{q}}$ a transition to the normal two-dimensional spin-glass behavior is found, i.e. $T_{\mathrm{c}}=0$.

The model consists of $N=L^{2}$ Ising spins $S_{i}= \pm 1$ on a square lattice with the Hamiltonian

$$
\mathcal{H}=-\sum_{\langle i, j\rangle} J_{i j} S_{i} S_{j}
$$

where the sum runs over all pairs of nearest neighbors $\langle i, j\rangle$ and the $J_{i j}= \pm J$ are quenched random variables.

The realizations are prepared exactly in the same way as in Ref. 12. For each realization, first $N$ bonds with strength $+J$ and $N$ bonds with strength $-J$ are distributed randomly among all $2 N$ bonds. Then the val- ues of all spins are set randomly to orientations $S_{i}= \pm 1$. Next, an annealed Monte-Carlo (MC) simulation ${ }^{14}$ is performed. This means, at each step either a spin is allowed to flip or two bonds incident to the same site are allowed to exchange their positions. Each choice occurs with probability 0.5. Each step is accepted with the usual Metropolis probability depending on the energy change according to the Hamiltonian (11). First, the system is equilibrated at high temperature $T=5$ for $1000 \mathrm{MC}$ steps per spin (MCS $)^{15}$. Finally the system is quenched to $T=T_{\mathrm{q}}$ and simulated for further 1000 MCS. The result is a realization of the disorder which can used for further treatment, here ground-state calculations are applied.

In greater than two dimensions, or in the presence of a magnetic field, the exact calculation of spin-glass ground states belongs to the class of NP-hard problems ${ }^{16.17}$. This means that only algorithms with exponentially increasing running time are known. However, for the special case of a planar system without magnetic field, e.g. a square lattice with periodic boundary conditions in at most one direction, there are efficient polynomial-time "matching" algorithms ${ }^{18}$. The basic idea is to represent each realization of the disorder by its frustrated plaquettes $\frac{19}{9}$. Pairs of frustrated plaquettes are connected by paths in the lattice and the weight of a path is defined by the sum of the absolute values of the coupling constants which are crossed by the path. A ground state corresponds the set of paths with minimum total weight, such that each frustrated plaquette is connected to exactly one other frustrated plaquette. This is called a minimum-weight perfect matching. The bonds which are crossed by paths connecting the frustrated plaquettes are unsatisfied in the ground state, and all other bonds are satisfied.

For the calculation of the minimum-weight perfect matching, efficient polynomial-time algorithms are available ${ }^{20.21}$. Recently, an implementation has been presented $^{22}$, where ground-state energies of large systems of size $N \leq 1800^{2}$ were calculated. Here, an algorithm from the LEDA library ${ }^{23}$ has been applied, which allows 
a quick implementation. It was not necessary to go beyond $N=256^{2}$ (with is much larger than $N=16^{2}$ in the original work ${ }^{12}$ ) to obtain reliable results.

To study whether an ordered phase is stable at finite temperatures, the following procedure is usually applied $^{2.3 .6 .7 .11 .24 .25}$. First a ground state of the system is calculated. Then the system is perturbed to introduce a domain wall and the new ground-state energy is evaluated. Typically, the system initially has periodic boundary conditions in both directions, and the perturbation involves replacing periodic by antiperiodic boundary conditions in one direction. The domain-wall energy $\Delta E$ is given by the difference of the two ground-state energies. In case the model exhibits long-range ferromagnetic order at non-zero temperatures, the domain-wall energy, averaged over many independent samples, has to increase with system size. E.g. for a pure $2 d$ ferromagnet, the domain wall consists of a straight line, resulting in $\Delta E \sim L$. For a spin-glass, none of the ground states with periodic and antiperiodic boundary conditions has a priori a lower energy. Hence one studies the absolute value of the domain-wall energy to detect whether the systems exhibits spin-glass ordering at finite temperatures.

However we cannot apply the matching algorithm for boundary conditions which "wrap around" in both directions. For this reason, here the periodic boundary conditions in the $\mathrm{y}$-direction are broken for each realization (by setting the bonds connecting the first and the last row to zero). This has no influence on the fact of whether the systems orders or not because the change of the boundary conditions to create the domain walls occurs in the $\mathrm{x}$-direction perpendicular to the open boundaries.

Here system sizes $L=4,6,8, \ldots 192,256$ are considered. For each size, 1000 independent realizations of the disorder were generated for quenching temperatures $T_{\mathrm{q}}=0.1,0.9,0.95,1.0$ and 1.5. Then ground states with periodic (P) and antiperiodic (AP) boundary conditions in $x$-direction were calculated using the exact matching algorithm, resulting in ground-state energies $E_{\mathrm{P}}^{0}$ resp. $E_{\mathrm{AP}}^{0}$. The change in the boundary conditions introduces a domain wall in each realization with energy $\Delta E=E_{\mathrm{AP}}^{0}-E_{\mathrm{P}}^{0}$.

First, we consider a very low quenching temperature $T_{\mathrm{q}}=0.1$, which was claimed in Ref. 12 to exhibit a spinglass transition at $T_{\mathrm{c}} / J=2.22(1)$ and no ferromagnetic order. In Fig. 11 the distribition $P(\Delta E, L)$ over the disorder of the domain-wall energies is displayed for different system sizes $L$. Clearly, the domain-wall energies grow strongly with system size, which is an indicator for ferromagnetic order. Below (c.f. Fig. 4) it is shown that indeed the disorder average $\langle\Delta E\rangle$ grows linearly with $L$, as in the normal ferromagnet. But the model exhibits no global magnetic moment, as found already in Ref. 12. This is due to fact that $50 \%$ of all bonds are antiferromagnetic. Nevertheless, the model behaves like a ferromagnet. The reason is that the bonds are distributed in the system such that only few frustrated plaquettes are present. Hence, each realization can be mapped via a lo-

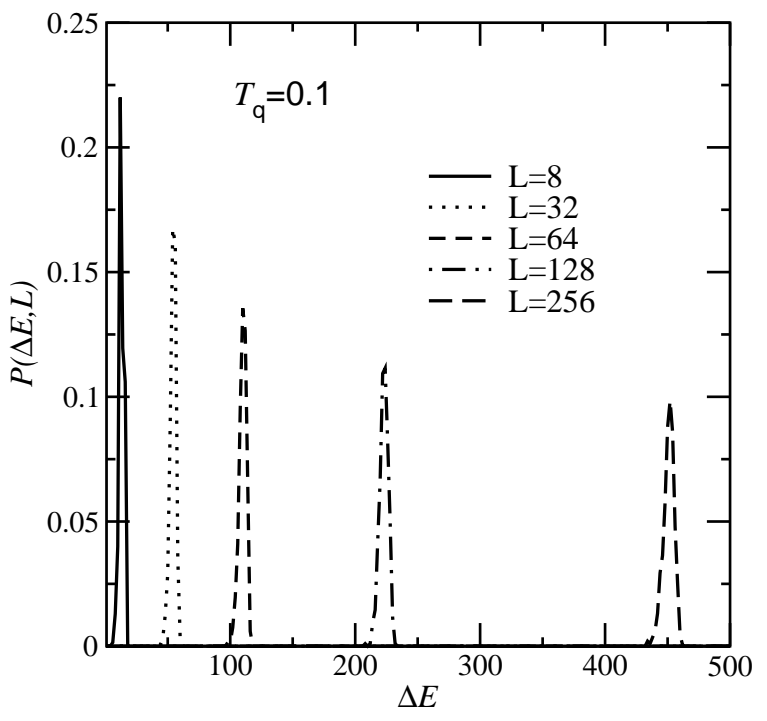

FIG. 1: Distribution $P(\Delta E, L)$ of domain-wall energies for $T_{\mathrm{q}}=0.1$ and system sizes $L=8,32,64,128,256$.

cal gauge transformation on a ferromagnet with a small number of antiferromagnetic bonds. This explains the fact that in Ref. 12 the critical exponent of the correlation length found at $T_{\mathrm{c}} / J=2.22$ was indeed that of the pure ferromagnet.

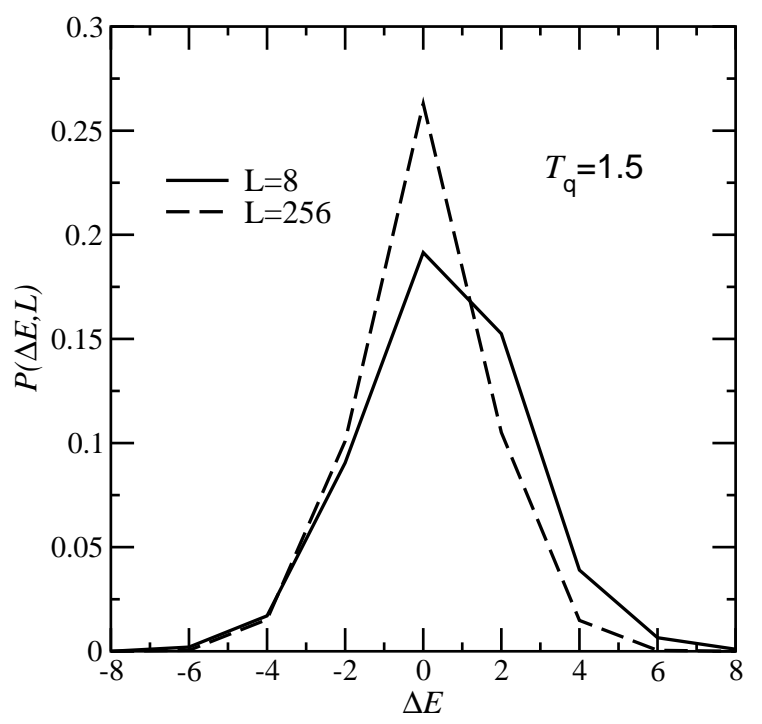

FIG. 2: Distribution of domain-wall energies for $T_{\mathrm{q}}=1.5$ and system sizes $L=8,256$.

Next, a large quenching temperature $T_{\mathrm{q}}=1.5$ is considered. In Fig. 2 again the distribution of domain-wall energies for different sizes are show. For large sizes, the distrubtions are centered around $\Delta E=0$ indicating the absence of ferromagnetic order. Furthermore, the width of the distributions decreases slightly with increasing system size, which shows that spin-glass order is not stable 
against thermal fluctuations. This is the usual situation found for the two-dimensional $\pm J$ spin glass 7.8 (having $\left.T_{\mathrm{c}} / J=0\right)$.

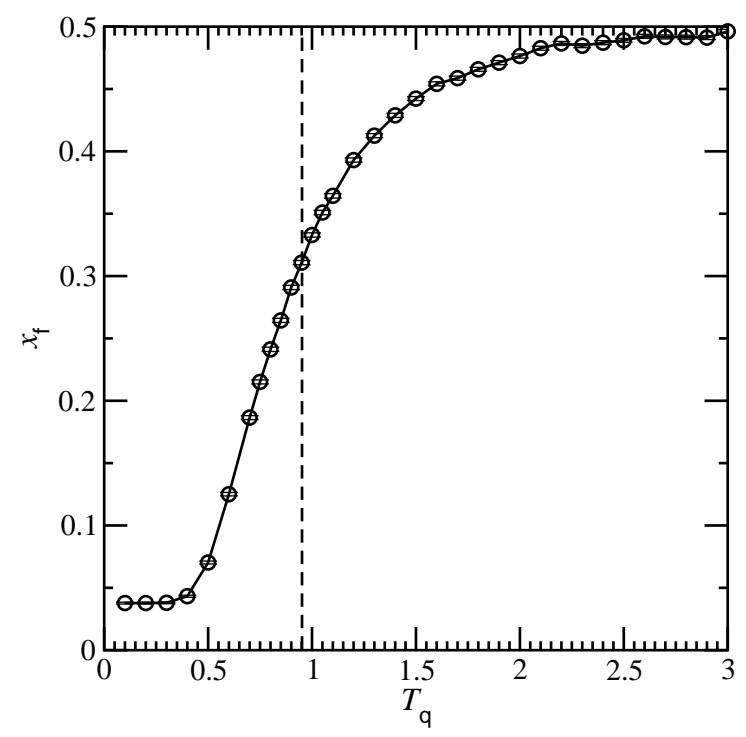

FIG. 3: Fraction of frustrated plaquettes present after the quench to temperature $T_{\mathrm{q}}$ as a function of $T_{\mathrm{q}}$. The line is a guide to the eyes only.

To understand the behavior of the system better, next it is analysed as a function of $T_{\mathrm{q}}$. The behavior is probably mainly determined by the fraction of frustrated plauqettes. In Fig. 3] the average fraction of frustrated plaquettes of the quenched realizations is shown as a function of $T_{\mathrm{q}}$. This can be compared with the standard $\pm J$ random bond model (with parameter $p \in[0,1]$ ), which has on average $2 N p$ antiferromagnetic and $2 N(1-p)$ ferromagnetic bonds. This results in a average fraction

$$
x_{\mathrm{f}}=4 x(1-x)\left[x^{2}+(1-x)^{2}\right]
$$

of frustrated plaquettes 26 . For the $\pm J$ model, a ferromagnet spin-glass $\left(T_{\mathrm{c}}=0\right)$ transition occurs $\$ .27 .28 .29 .30$ near $p=0.11$ were $x_{f}(0.11) \approx 0.31$. This corresponds to a quenching temperature $T_{\mathrm{q}} \approx 0.95$, see Fig. 3 Hence, for a comparison, simulations near $p=0.11$ for the $\pm J$ model and near $T_{\mathrm{q}} \approx 0.95$ for the "mobile-bond" model have been performed. Furthermore both models were investigated for two other pairs of parameters exhibiting similar concentrations of frustrated plaquettes: $T_{\mathrm{q}}=0.9 ; p=0.1$ and $T_{\mathrm{q}}=1.0 ; p=0.12$.

In Fig. 4 the mean value $\langle\Delta E\rangle$ of the domain-wall energy is shown as a function of the system size for $T_{\mathrm{q}}=0.1,0.9,0.95,1.0$. For $T_{\mathrm{q}}=0.1$ a clear linear increase occurs, corresponding to a normal ferromagnet. For $T_{\mathrm{q}}=0.9$ the domain-wall energy still increases with system size. The resulting values are very similar to the domain-wall energies found at $p=0.1$ for the $\pm J$ model.

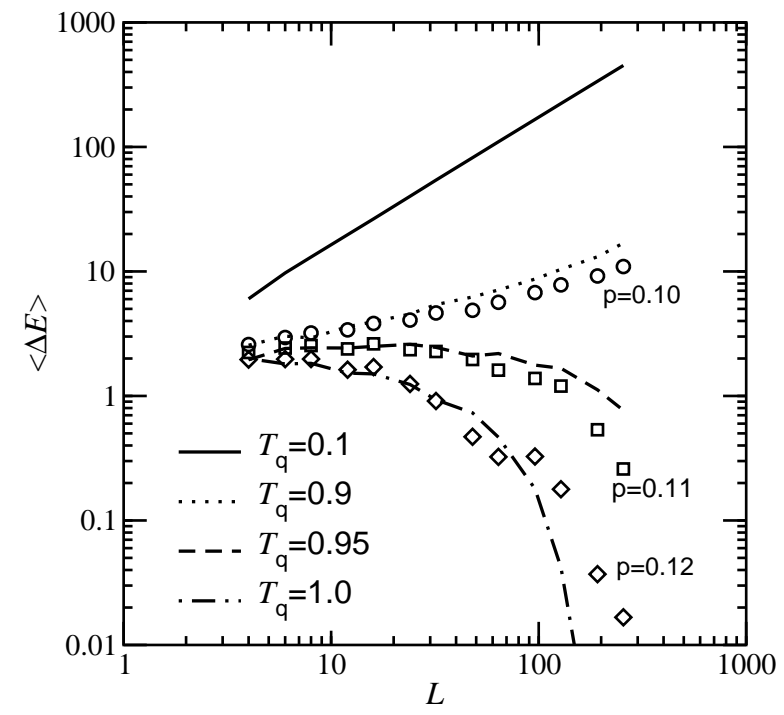

FIG. 4: Mean value of the domain-wall energy $\langle\Delta E\rangle$ as a function of system size for $T_{\mathrm{q}}=0.1,0.9,0.95,1.0$ (displayed by lines). Also the domain-wall energy for the $\pm J$ randombond model with concentrations $p=0.1,0.11,0.12$ of the antiferromagnetic bonds is shown (symbols).

For $T_{\mathrm{q}}=0.95$ and $T_{\mathrm{q}}=1$ the mean domain-wall energies decrease as a function of the system size, hence no ferromagnetic order persists. In these cases the data is almost equal to the results for $p=0.11$ resp. $p=0.12$ of the $\pm J$ model. Please note that it is not claimed here that e.g. $T_{\mathrm{q}}=0.9$ corresponds exactly to $p=0.1$. But it seems certainly possible to chose $p$ such that the results for both models agree exactly.

In Fig. 5 the corresponding results for the mean $\langle|\Delta E|\rangle$ of the absolute value of the domain-wall energy is shown. For values $T_{\mathrm{q}} \leq 0.9$ again an increase is observed, due to the increase of the mean (non absolute) $\langle\Delta E\rangle$. If spinglass ordering existed in a system, then $\langle|\Delta E|\rangle$ would increase with growing $L$, while $\langle\Delta E\rangle$ has to decrease. For $T_{\mathrm{q}}=0.95,1.0\langle|\Delta E|\rangle$ increases only for small system sizes (which may cause signs of a stable spin-glass phase when simulating only small systems), while it starts to decrease with $L$ for larger values of $L$. Hence spin-glass order is destroyed for any finite temperature $T>0$. Please note that again the results at $T_{\mathrm{q}}=0.9,0.95,1.0$ agree well with the results at $p=0.10,0.11,0.12$ for the $\pm J$ model.

To conclude, in this work the recently proposed "mobile-bond" spin-glass model has been investigated. An exact ground-state matching algorithm has been applied, allowing to study large system sizes like $N=256^{2}$. The model turns out to be mainly equivalent to the $\pm J$ random-bond model, which has been studied extensively in the past. Hence, for low values of the annealing temperature $T_{\mathrm{q}}$, the model (corresponding to small concentrations $p$ of the antiferromagnetic bonds in the $\pm J$ model) exhibits ferromagnetic order. The only difference is that the Sunko model exhibits no magnetic moment, 


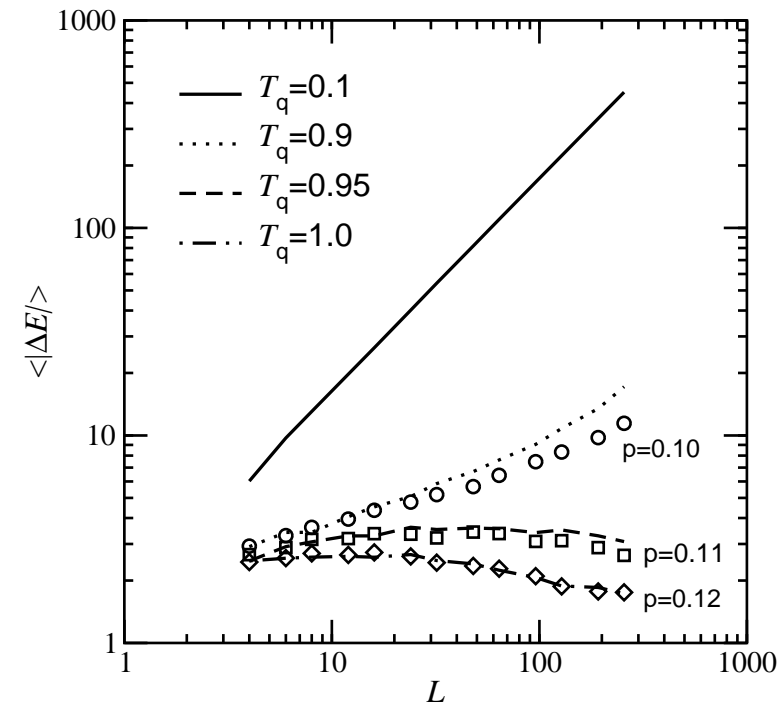

FIG. 5: Mean absolute value of the domain-wall energy $\langle|\Delta E|\rangle$ as a function of system size for $T_{\mathrm{q}}=0.1,0.9,0.95,1.0$ (displayed by lines). Also the absolute value of the domainwall energy for the $\pm J$ random-bond model with concentrations $p=0.1,0.11,0.12$ of the antiferromagnetic bonds is shown (symbols). since by construction the number of ferromagnetic bonds equals the number of antiferromagnetic bonds. Both models can be mapped onto each other by local gaugetransformations, the characteristic parameter is the fraction of frustrated plaquettes.

For larger values of $T_{\mathrm{q}} \geq 0.95$ (corresponding to $p \geq$ 0.11 ) the model displays the standard behavior of a twodimensional $\pm J$ spin-glass, hence no order for $T>0$ exists. To summarize, the "mobile-bond" model does not exhibit a finite-temperature spin-glass transition at any value of $T_{\mathrm{q}}$, opposed to the claims made in Ref ${ }^{12}$.

Acknowledgements: The author obtained financial support from the VolkswagenStiftung (Germany) within the program "Nachwuchsgruppen an Universitäten". He thanks A.P. Young for critically reading the manuscript. The simulations were performed on a Linux cluster at the Gesellschaft für wissenschaftliche Datenverarbeitung Göttingen (GWDG) (Germany).
1 Reviews on spin glasses can be found in: K. Binder and A.P. Young, Rev. Mod. Phys. 58, 801 (1986); M. Mezard, G. Parisi, M.A. Virasoro, Spin glass theory and beyond, (World Scientific, Singapore 1987); K.H. Fisher and J.A. Hertz, Spin Glasses, (Cambridge University Press, Cambridge 1991); A.P. Young (ed.), Spin glasses and random fields, (World Scientific, Singapore 1998).

2 W.L. McMillan, Phys. Rev. B 30, 476 (1984).

3 A.J. Bray and M.A. Moore, J. Phys. C 17, L463 (1984).

${ }^{4}$ T. Shirakura and F. Matsubara, Phys. Rev. Lett. 79, 2887 (1997).

${ }^{5}$ F. Matsubara, T. Shirakura, and M. Shiomi, Phys. Rev. B 58, R11821 (1998).

${ }^{6}$ H. Rieger, L. Santen, U. Blasum-U, M. Diehl, M. Jünger, and G. Rinaldi, J. Phys. A 29, 3939-50 (1996).

7 N. Kawashima and H. Rieger, Europhys. Lett. 39, 85 (1997).

8 A.K. Hartmann and A.P. Young, Phys. Rev B 64, 180404 (2001).

9 J. Houdayer Eur. Phys. J. B 22, 479 (2001).

10 A.C. Carter, A.J. Bray, and M.A. Moore, Phys. Rev. Lett. 88, 077201 (2002).

11 A.K. Hartmann and I.A. Campbell, Phys. Rev. B 63, 094423 (2001).

12 D.K. Sunko, preprint cond-mat/0212346 (2002); P. Lazić and D.K. Sunko, Eur. J. Phys. B 21, 595 (2001).

13 J. R. Banavar and M. Cieplak, Phys. Rev. Lett. 48, 832 (1982).

14 D.P. Landau and K. Binder, A Guide to Monte Carlo Simulations in Statistical Physics, (Cambridge University
Press, Cambridge 2000).

15 The time to equilibrate the system is only few MC steps per spin. This has been checked by starting with fully frustrated and fully unfrustrated systems and measuring the level of frustration and the energy as a function of the number of MC steps.

16 F. Barahona, J. Phys. A 15, 3241 (1982).

17 A.K. Hartmann and H. Rieger, Optimization Algorithms in Physics, (Wiley-VCH, Berlin, 2001).

18 I. Bieche, R. Maynard, R. Rammal, and J.P. Uhry, J. Phys. A 13, 2553 (1980).

19 G. Toulouse, Commun. Phys. 2, 115 (1977).

20 F. Barahona, R. Maynard, R. Rammal, and J.P. Uhry, J. Phys. A 15, 673 (1982).

21 U. Derigs and A. Metz, Math. Prog. 50, 113 (1991).

22 R.G. Palmer and J. Adler, Int. J. Mod. Phys. C 10, 667 (1999).

${ }^{23}$ K. Mehlhorn and St. Näher, The LEDA Platform of Combinatorial and Geometric Computing, Cambridge University Press, Cambridge 1999; see also http://www.mpi-sb.mpg.de/LEDA/leda.html

24 A.K. Hartmann, Phys. Rev. E 59, 84 (1999).

25 A.K. Hartmann, Phys. Rev. E 60, 5135 (1999).

26 S. Kirkpatrick, Phys. Rev. B 16, 4630 (1977).

27 G. Grinstein, C. Jayaprakash and M. Wortis, Phys. Rev. B 19, 260 (1979).

${ }^{28}$ H. Freund and P. Grassberger, J. Phys. A 22, 4045 (1989).

29 J. Bendisch, J. Stat. Phys. 67, 1209 (1992).

${ }^{30}$ F. Merz and J.T. Chalker, Phys. Rev. B 65, 054425 (2002). 